\title{
Nonlinear Optical Properties of a MMA-Silica Nanohybrid Material Doped with Rhodamine 6G
}

\author{
J. Lima-Gutiérrez, ${ }^{1,2,3}$ R. Palomino-Merino, ${ }^{1,2}$ M. L. Arroyo Carrasco, ${ }^{1,2}$ \\ E. Rubio-Rosas, ${ }^{2,4}$ and V. M. Castaño ${ }^{5}$ \\ ${ }^{1}$ Facultad de Ciencias Físico Matemáticas, Avenida San Claudio y 18 Sur, Colonia San Manuel, 72570 Pubela, PUE, Mexico \\ ${ }^{2}$ Benemérita Universidad Autónoma de Puebla Ciudad Universitaria, Colonia San Manuel, 72570 Pubela, PUE, Mexico \\ ${ }^{3}$ Departamento de Ciencias e Ingenierías, Universidad Iberoamericana Puebla, Boulevard del Niño Poblano No. 2901, \\ Unidad Territorial Atlixcáyotl, 72197 Pubela, PUE, Mexico \\ ${ }^{4}$ Centro Universitario de Vinculación y Transferencia de Tecnología, Prolongación de la 24 Sur y Avenida San Claudio, \\ Ciudad Universitaria, Colonia San Manuel, 72570 Puebla, PUE, Mexico \\ ${ }^{5}$ Centro de Física Aplicada y Tecnología Avanzada, UNAM, A.P. 1-1010, 76000 Querétaro, QRO, Mexico
}

Correspondence should be addressed to V. M. Castaño; meneses@unam.mx

Received 4 June 2013; Accepted 28 November 2013

Academic Editor: Sudhakar Nori

Copyright ( 2013 J. Lima-Gutiérrez et al. This is an open access article distributed under the Creative Commons Attribution License, which permits unrestricted use, distribution, and reproduction in any medium, provided the original work is properly cited.

\begin{abstract}
A novel nanohybrid material based on MMA-Silica has been synthesized with an organic dye dopant (R6G) to tailor the optical properties. This novel material can be used on several devices such as active laser media for an organic solid state laser, OLEDs, or as a characterization media for new organic dye molecules. Thin films were deposited by dip-coating and characterized by absorption and reflection UV-VIS, photoluminescence, SEM, and Z-scan technique to verify their nonlinear behavior. R6G dye dopant has been used to verify that the nanohybrid matrix does not inhibit its optical properties.
\end{abstract}

\section{Introduction}

There is a need for developing new economical and simple materials suitable for different applications, in particular those related to nonlinear optical behavior, for applications ranging from nanomedicine to nanoelectronics. For instance, when a nonlinear material is doped with an organic dye it can be used as a laser medium for solid-state organic dye lasers. Nowadays solid-state polymeric dye lasers are of interest [1-3]; they can be used in medicine and cosmetology [4$6]$, optics $[7,8]$, electronics $[9,10]$, and other disciplines. New ways to generate laser light have been developed; tunable solid-state lasers can be obtained by the incorporation of guest dyes in solid host materials and this is one of the ideas that motivates this work. So far, several lightemitting solid systems based on the inclusion of laser dyes inside organic and inorganic solid matrix and hybrid and nanohybrid materials have been developed [11]. The use of sol-gel technique [12] allows incorporating dyes in a solid material at the nanoscale; this technique is based on the hydrolysis of a precursor, forming a gel, and in this part of the process a dopant can be added. When solvents are volatilized a nanoporous glass with the inclusion of the dopant is obtained. So this technique is important because it can change optical, mechanical, thermal, and electrical properties of the matrix formed by the precursor by adding different kinds of dopants like powders, liquids, organic and inorganic materials, nanoparticles, and so forth. The dopants chosen depends on the required material properties and the changes on the amount of dopant inside the matrix. For example, it is possible to add an organic dye to have specific optical properties and some polymer to change its mechanical properties, like flexibility, or to protect the organic dye; PMMA (polymethylmethacrylate) decreases organic dyes degradation on solid-state dye lasers. OLEDs $[13,14]$ are another technology where organic dyes are used 
and can be created with sol-gel technique adding dopants like R6G (rhodamine 6G), an element of a family of dye molecules used in a variety of applications. As a dye it can be used in staining materials or as a tracer in flow and speed determinations of water or other solvents. It possesses a characteristic absorbance spectrum which can be easily detected. In addition R6G fluoresces with high efficiency making it an invaluable component of a dye laser which can be pumped by the second harmonic of a Nd:YAG laser $(\lambda=532 \mathrm{~nm})$; for this purpose its very important to study its photobleaching features in order to know how it degrades under the incident pump laser; this is achieved by doping PMMA with R6G [15]. The optical properties of a hybrid material can be tailored doping it with several dyes in order to obtain a variety of emission lines; this could be of interest in the manufacture of dye lasers [16].

\section{Experimental}

The sol-gel technique is characterized by many of its advantages such as simplicity of the synthesis, facility to be prepared at room temperature, and convenience to be doped with different materials like powders or liquids. This is important because dopant can change the optical or mechanical properties of the material, at the nanoscale, for example, by adding PMMA to a nanoporous silica matrix improving a more flexible material in comparison to a pure nanoporous silica matrix that is hard. We choose silica and PMMA as precursors because both are transparent materials at visible region so they do not change the optical properties of organic dyes and R6G was chosen for its well-known UV-Vis spectra, so R6G:ethanol spectrum can be compared with Hyb:R6G to check for changes. Deep-coating technique is used to grow thin films on glass substrates.

For the synthesis of this nanohybrid material [17], by sol-gel technique two steps are required; reaction was initiated with MMA and some pills of $\mathrm{NaOH}$ in a clean flask; the reaction was allowed to proceed for $15 \mathrm{~min}$. In a second glass MMA, TEOS (tetraethyl orthosilicate), TMSPM, ethanol, and water are poured with molar ratio MMA: TEOS : TMSPM : ethanol : water of $3: 1: 1: 15: 15$. Finally both containers are poured into one glass and drops of $\mathrm{HCl}$ are added to obtain a $\mathrm{pH} 4$. It was stirred for 24 hours. All reactions occurred at room temperature. R6G was used as dopant with a molar ratio TEOS:R6G of 1:0.001; that is, the molar amount $(\nu)$ of R6G added to the hybrid matrix is given by $v=0.001 \nu_{\text {TEOS }} N$, where $\nu_{\text {TEOS }}$ are the moles of TEOS and $N$ the concentration number of $\mathrm{R} 6 \mathrm{G}$ which goes from $N=0$ not dopant, $N=1$ the first concentration of R6G, and so on until saturation.

Thin films were deposited using deep-coating technique on glass substrates previously cleaned in piranha solution, rinsed in ethanol, and dried at room temperature. Thin film thickness is controlled by fixing the substrate deposition extraction rate and speed.

A Spectronic Unicam UV 300 spectrometer was used to measure absorption and reflectance UV-Vis; photoluminescence was obtained using a B\&W TEK $407 \mathrm{~nm}$ violet solid state laser $3.04 \mathrm{eV}, 60 \mu \mathrm{W}$, Sciencetech 9040 monochromator of $0.5 \mathrm{~m}$ optical path. For the $Z$-scan technique was used a He:Ne laser as light source, $\lambda=633 \mathrm{~nm}$ of $10 \mathrm{~mW}$ of power; SEM micsrographs were taken in a JEOL JSM-6610.

\section{Results and Discussion}

Figure 1 contains the IR-transmission spectrum of nanohybrid matrix and nanohybrid matrix doped with R6G; no mayor differences were found because R6G does not change the nanohybrid matrix. We can find bands representing the nanoporous silica matrix at 1194 and $939 \mathrm{~cm}^{-1}$ for $\mathrm{Si}-\mathrm{O}-\mathrm{Si}$ and $\mathrm{Si}-\mathrm{OH}$ asymmetric stretching vibrations, respectively, also among those bands can find one related to $\mathrm{Si}-\mathrm{O}-\mathrm{CH}_{3}$; and a peak at $787 \mathrm{~cm}^{-1}$ related to $\mathrm{Si}-\mathrm{O}-\mathrm{Si}$ symmetrical vibrations. Some other bands related to PMMA are found at $2351 \mathrm{~cm}^{-1}$ and $2912 \mathrm{~cm}^{-1}$. This indicates that the nanoporous silica matrix and the polymeric matrix are independent of each other; they are related only by a bending vibration at $1457 \mathrm{~cm}^{-1}$ due to TMSPM. The aliphatic chain $-\mathrm{CH}-$ is revealed through a stretching band at $2959 \mathrm{~cm}^{-1}$. Absorbed water band is at $3350 \mathrm{~cm}^{-1}$. A weak $\mathrm{C}=\mathrm{C}$ peak at $1700 \mathrm{~cm}^{-1}$ is due to unpolymerized MMA; $\mathrm{CH}_{2}$ band is at $2926 \mathrm{~cm}^{-1}$ and an aliphatic chain - $\mathrm{CH}-$ at $2840 \mathrm{~cm}^{-1}$.

Beer-Lambert law $(A=m c t, m$ molar absorbance coefficient) shows that absorbance $A$ increases when dopant concentration $c$ or sample thickness $t$ is increased. That behavior is observed in Figure 2, where we plot thin films absorption spectra of the nanohybrid material at different concentrations of R6G, without R6G $(N=0)$, with a first concentration of R6G $(N=1)$, and so forth. When R6G concentration is increased the absorbance increases. Another way to change the absorbance is by changing the film thickness; however, in all the samples used here thickness remains constant because the speed of immersion and extraction of the substrates were fixed.

R6G is absorbed in the visible spectrum between $440 \mathrm{~nm}$ and $580 \mathrm{~nm}$, corresponding to frequencies between the end of violet and half yellow having a maximum absorption for the green at $532 \mathrm{~nm}$ (Figure 2); a secondary maximum is shown at $490 \mathrm{~nm}$, so R6G absorption spectrum profile is the convolution of two absorptions. The same profile is generated with ethanol:R6G. The authors Lu and Penzkofer [18] determine the optical properties of R6G dissolved in methanol and of a solid R6G film finding deviations from Beer's law due to the mutual interactions of neighbouring molecules and explain what the main and secondary peak are due for the generation of monomers and dimmers of R6G. R6G dissolved in methanol shows a peak at $530 \mathrm{~nm}$, due to monomers and a secondary peak at $490 \mathrm{~nm}$ due to dimmers or multimers; monomers population is higher than dimmers. In the case of the hybrid material ethanol is part of the medium and during the hydrolysis reaction ethanol is produced so $\mathrm{R} 6 \mathrm{G}$ immersed in the hybrid matrix has the same behavior as in methanol. This is the reason why the absorbance spectrum is maintained; on the other hand PMMA and $\mathrm{SiO}_{2}$ are transparent at visible region of the spectrum so they do not have any optical contribution to the dye absorbance. 


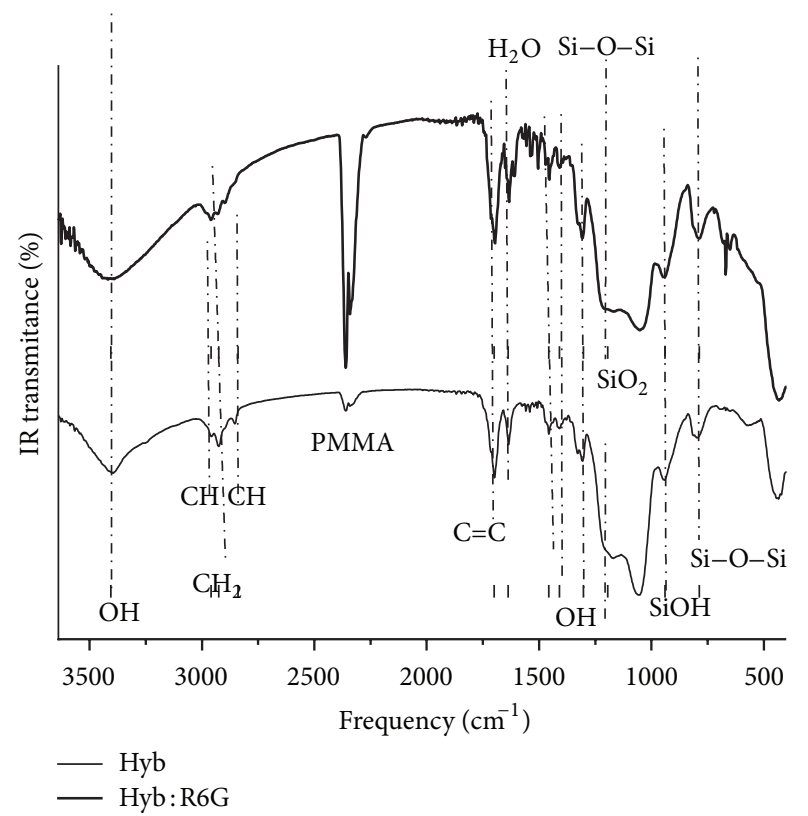

FIGURE 1: IR transmittance for hybrid material and Hybrid:R6G. Silica matrix is represented by $\mathrm{Si}-\mathrm{O}-\mathrm{Si}, \mathrm{SiO}_{2}$ bounds; polymeric matrix peak is at $2351 \mathrm{~cm}^{-1}$ and $2912 \mathrm{~cm}^{-1}$.

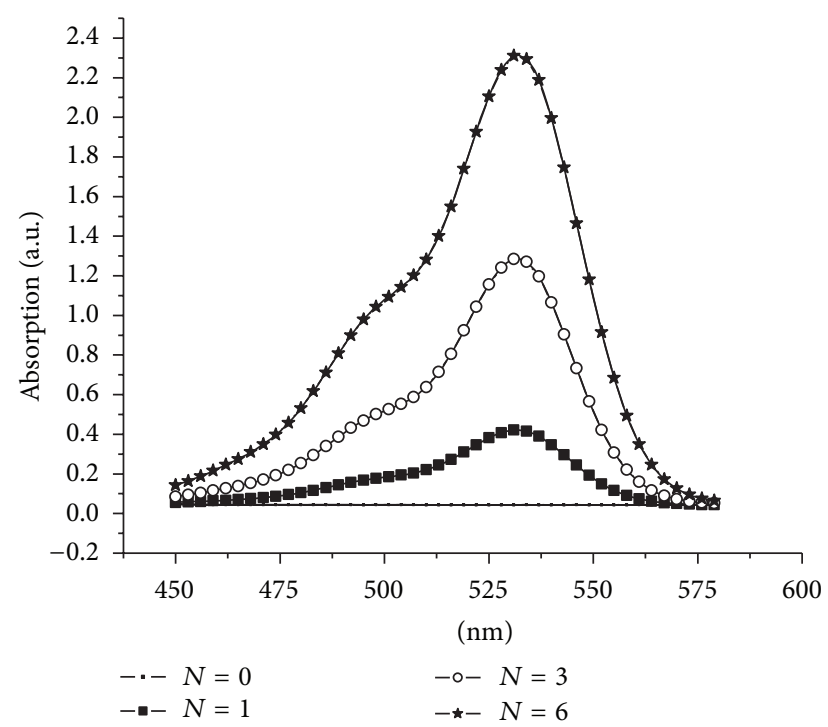

FIGURE 2: Hybrid material does not present absorption at visible spectra; when dopant of R6G is increased absorption response is increased according to Beer-Lambert law; this spectrum is characteristic for the R6G where the principal peak is around $532 \mathrm{~nm}$.

Figure 3 shows the reflectance spectrum of thin films with different concentrations at $30^{\circ}$; when the R6G concentration increases the reflectance of the samples between $450 \mathrm{~nm}$ and $590 \mathrm{~nm}$ decreases and broadens; it is due to the absorbance at $532 \mathrm{~nm}$; this behavior is useful for a device in which we need to have a filter for those frequencies; the control is generated through the concentration of R6G.

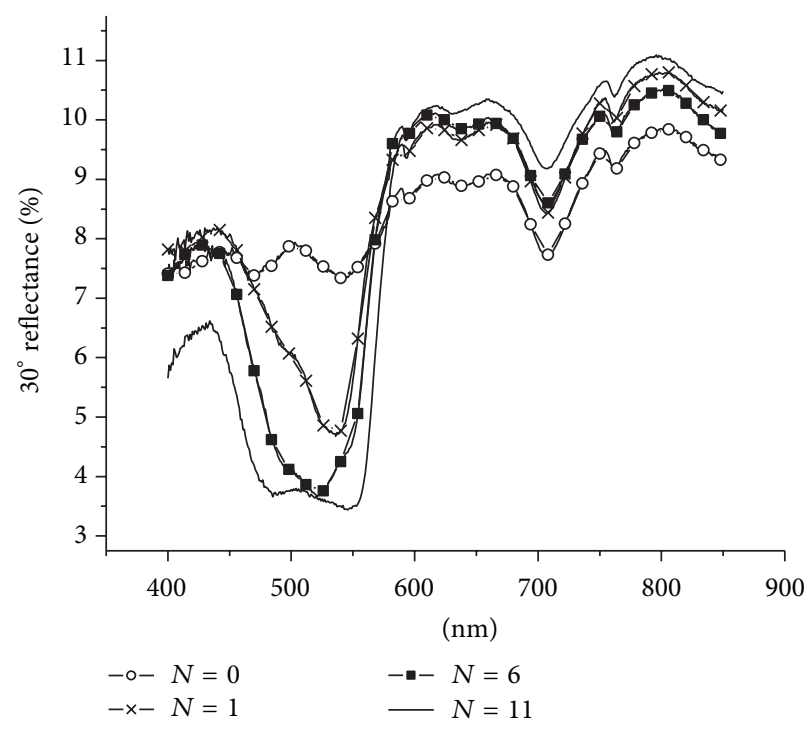

FIGURE 3: Reflectance spectra of the samples without dopant, first concentration of rhodamine $6 \mathrm{G}$, and sixth and eleventh concentration measured at 30 degrees; the higher concentration decreases the reflectance between $450 \mathrm{~nm}$ and $590 \mathrm{~nm}$.

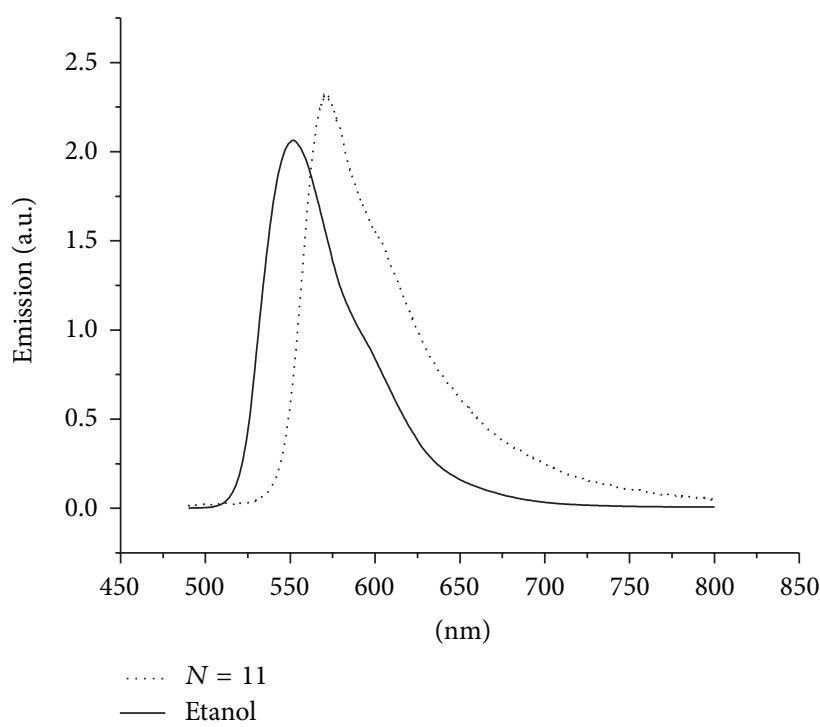

FIGURE 4: Photoluminescence spectra of Hyb:R6G for the eleventh concentration compared to the emission of $\mathrm{R} 6 \mathrm{G}$ dissolved in ethanol; both spectra are similar.

Photoluminescence is the emission of light from a material if their atoms were excited by absorption of incident light; when they decay into lower energy states part of the absorbed energy is emitted as photons of less energy than the incidents. Luminescence can be emitted by organic or inorganic compounds. Figure 4 shows the photoluminescence spectrum of the hybrid material doped with R6G showing a maximum luminescence at $571.8 \mathrm{~nm}$, and a secondary at $600 \mathrm{~nm}$; these correspond to the emissions for the absorbed photons at $532 \mathrm{~nm}$ and $590 \mathrm{~nm}$, respectively, which is very close to that reported when R6G is immersed in ethylene 


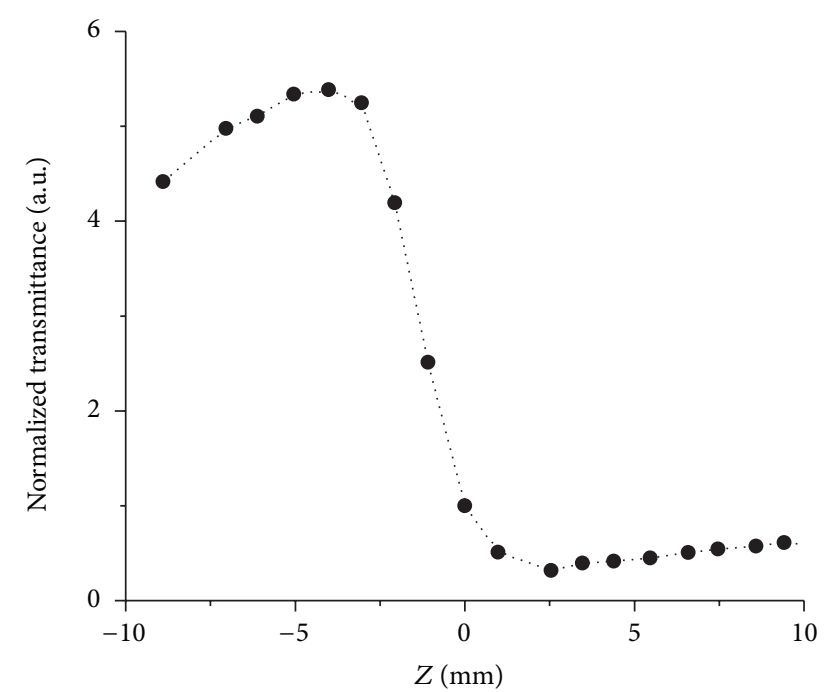

FIGURE 5: $Z$-scan curve of R6G dissolved in ethanol immersed in the nanohybrid material. Nonlinear behavior is due to the rhodamine $6 \mathrm{G}$ molecule. Hybrid material does not inhibit the nonlinear behavior.

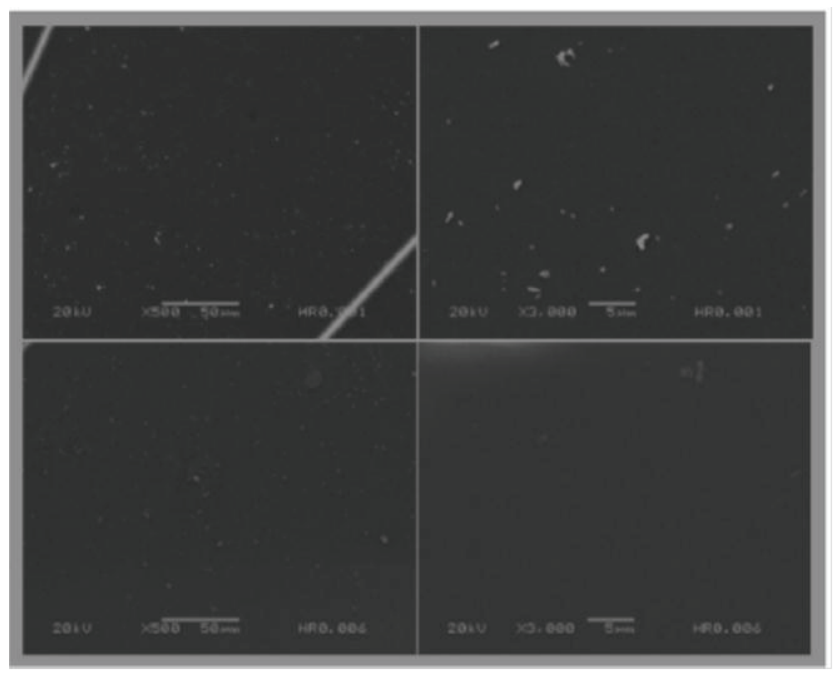

FIGURE 6: Micrographs of thin films with the first (first row) and sixth (second row) concentrations of R6G, at 500x (first column) and 3000x (second column); none of the films show fractures.

glycol [19], where a fluorescence peak is at $578.2 \mathrm{~nm}$ and when R6G is immersed in ethanol where the maximum photoluminescence is at $551 \mathrm{~nm}$. So we can argue that the hybrid matrix does not radically change the wavelength of fluorescence of R6G; it is important to notice that hybrid matrix does not present photoluminescence so the observed spectrum is due solely to R6G photoluminescence spectra; the reason that the hybrid matrix does not show emission is because $\mathrm{SiO}_{2}$ and MMA were not absorbed at visible region. To know photoluminescence spectra can be of interest to develop a solid-state dye laser [20]. A scintillator is a material used for detection and monitoring of accelerated particles like cosmic rays, among others; R6G is a dye used for scintillators due to its fluorescence emission properties [21].
Not only with R6G optical absorption and fluorescence properties are maintained but also with any organic dye dissolved in ethanol, so the same hybrid matrix can be doped with several organic dyes at the same time maintaining their optical properties. For many years it has been known that some kinds of organic materials exhibit extremely large nonlinear optical and electrooptical responses. The electronic nonlinearities in the most efficient organic materials are essentially based on molecular units containing highly delocalized $\pi$-electrons and additional electron-donor and electron-acceptor groups on opposite sides of the molecules. The highly active molecules tend to be highly polarized. The most polar molecules have a tendency to crystallize in a centrosymmetric structure which does not show nonlinear optical effects of second order. The choice of special molecules and the crystal growth methods used are of main importance for obtaining electrooptically active single crystals of good quality and large size. A main advantage of the organic materials is the possibility of altering the molecular structure for optimizing the electrooptical or nonlinear optical properties.

Figure 5 shows the $Z$-scan curve for a sample of R6G dissolved in ethanol immersed in the nanohybrid material, using as light source a He:Ne laser, with an intensity of $790 \mathrm{~W} / \mathrm{cm}^{2}$ in the material, $\lambda=633 \mathrm{~nm}$. The sample exhibits a negative nonlinear optical response. This nonlinear behavior is due to the presence of R6G in the hybrid material which does not show a nonlinear optical response so nonlinear curve shown in Figure 5 is only due to R6G dissolved in ethanol, hybrid matrix does not inhibit its nonlinear response which can be modified by increasing dopant quantity or adding different organic dyes.

Different samples were observed by SEM showing that during the drying process of thin films solvents are volatilized breaking the films, but these films do not have fractures (Figure 6) because the polymer matrix is flexible so that the fractures are reduced owing to the matrix by which is opened allowing the passage of solvents.

\section{Conclusions}

A simple and inexpensive optically nonlinear nanohybrid material, based on a TEOS:MMA nanoporous matrix, was developed. Optical response control depends on the thin film thickness or on the amount of dopant; these results can be seen using R6G as reference. The TEOS:MMA hybrid matrix does not change the absorbance and photoluminescence spectra of the organic dopant because TEOS and PMMA are transparent at visible spectra. Sol nanoparticles of the hybrid material doped with an organic dye show optical nonlinear response due to the dopant. Some potential applications for this material include lasers, photochromic coatings, and photoluminescent materials for organic devices and biomedical imaging.

\section{References}

[1] Y. Li, M. Sasaki, and K. Hane, "Fabrication and testing of solid polymer dye microcavity lasers based on PMMA 
micromolding," Journal of Micromechanics and Microengineering, vol. 11, no. 3, p. 234, 2001.

[2] S. Singh, V. R. Kanetkar, G. Sridhar, V. Muthuswamy, and K. Raja, "Solid-state polymeric dye lasers," Journal of Luminescence, vol. 101, no. 4, pp. 285-291, 2003.

[3] D. Schneider, S. Hartmann, T. Benstem et al., "Wavelengthtunable organic solid-state distributed-feedback laser," Applied Physics B, vol. 77, no. 4, pp. 399-402, 2003.

[4] B. R. Moody, J. E. McCarthy, G. J. Hruza, and M. Alam, "Collagen remodeling after 585-nm pulsed dye laser irradiation: an ultrasonographic analysis," Dermatologic Surgery, vol. 29, no. 10, pp. 997-1000, 2003.

[5] M. R. Alexiades-Armenakas, J. S. Dover, and K. A. Arndt, "The spectrum of laser skin resurfacing: nonablative, fractional, and ablative laser resurfacing," Journal of the American Academy of Dermatology, vol. 58, no. 5, pp. 719-737, 2008.

[6] M. Lapidoth, M. E. Y. Odo, and L. M. Odo, "Novel use of erbium: YAG $(2,940-\mathrm{nm})$ laser for fractional ablative photothermolysis in the treatment of photodamaged facial skin: a pilot study," Dermatologic Surgery, vol. 34, no. 8, pp. 1048-1053, 2008.

[7] R. Bornemann, U. Lemmer, and E. Thiel, "Continuous-wave solid-state dye laser,” Optics Letters, vol. 31, no. 11, pp. 1669-1671, 2006.

[8] T. Li, Z. Zhuo, X. Li, H. Yang, and Y. Zhang, "Study on optical characteristics of Nd:YVO4/YVO4 composite crystal laser," Chinese Optics Letters, vol. 5, no. 3, pp. 175-177, 2007.

[9] A. Costela, I. García-Moreno, and R. Sastre, "Polymeric solidstate dye laser: recent developments," Physical Chemistry Chemical Physics, vol. 5, no. 21, pp. 4745-4763, 2003.

[10] G. Wu, C. Li, Y. Liu, N. Dai, and J. Wang, "Study on high resolution time interval measurement module in pulsed laser ranging system," Journal of Infrared and Millimeter Waves, vol. 26, no. 3, pp. 213-221, 2007.

[11] C. Sanchez, B. Julián, P. Belleville, and M. Popall, "Applications of hybrid organic-inorganic nanocomposites," Journal of Materials Chemistry, vol. 15, no. 35-36, pp. 3559-3592, 2005.

[12] C. J. Brinker and G. W. Scherer, Sol-Gel Science: The Physics and Chemistry of Sol-Gel Processing, Academic Press, 1990.

[13] B. Choudhury, R. Shinar, and J. Shinar, "Glucose biosensors based on organic light-emitting devices structurally integrated with a luminescent sensing element," Journal of Applied Physics, vol. 96, no. 5, pp. 2949-2954, 2004.

[14] A. Pais, A. Banerjee, D. Klotzkin, and I. Papautsky, "Highsensitivity, disposable lab-on-a-chip with thin-film organic electronics for fluorescence detection," Lab on a Chip, vol. 8, no. 5, pp. 794-800, 2008.

[15] N. A. George, B. Aneeshkumar, P. Radhakrishnan, and C. P. G. Vallabhan, "Photoacoustic study on photobleaching of Rhodamine 6G doped in poly(methyl methacrylate)," Journal of Physics D, vol. 32, no. 14, pp. 1745-1749, 1999.

[16] D. Moses, "High quantum efficiency luminescence from a conducting polymer in solution: a polymer laser dye," Synthetic Metals, vol. 55, no. 1, pp. 22-27, 1993.

[17] E. Rubio, J. Almaral, R. Ramirez-Bon, V. Castaño, and V. Rodríguez, "Organic-inorganic hybrid coating (poly(methyl methacrylate)/monodisperse silica)," Optical Materials, vol. 27, pp. 1266-1269, 2005.

[18] Y. Lu and A. Penzkofer, "Absorption behaviour of methanolic rhodamine 6G solutions at high concentration," Chemical Physics, vol. 107, no. 2-3, pp. 175-184, 1986.
[19] N. Sharma, N. Singh, H. S. Vora, and S. L. Goyal, "Influence of the medium on the fluorescence of copper vapor laser pumped rhodamine 6G dye: addendum," Optics Journal, vol. 1, pp. 18-22, 2007.

[20] R. Sasai, N. Iyi, T. Fujita et al., "Luminescence properties of rhodamine $6 \mathrm{G}$ intercalated in surfactant/clay hybrid thin solid films," Langmuir, vol. 20, no. 11, pp. 4715-4719, 2004.

[21] M. Nikl, N. Solovieva, K. Apperson, D. J. S. Birch, and A. Voloshinovskii, "Scintillators based on aromatic dye molecules doped in a sol-gel glass host," Applied Physics Letters, vol. 86, no. 10, Article ID 101914, 3 pages, 2005. 

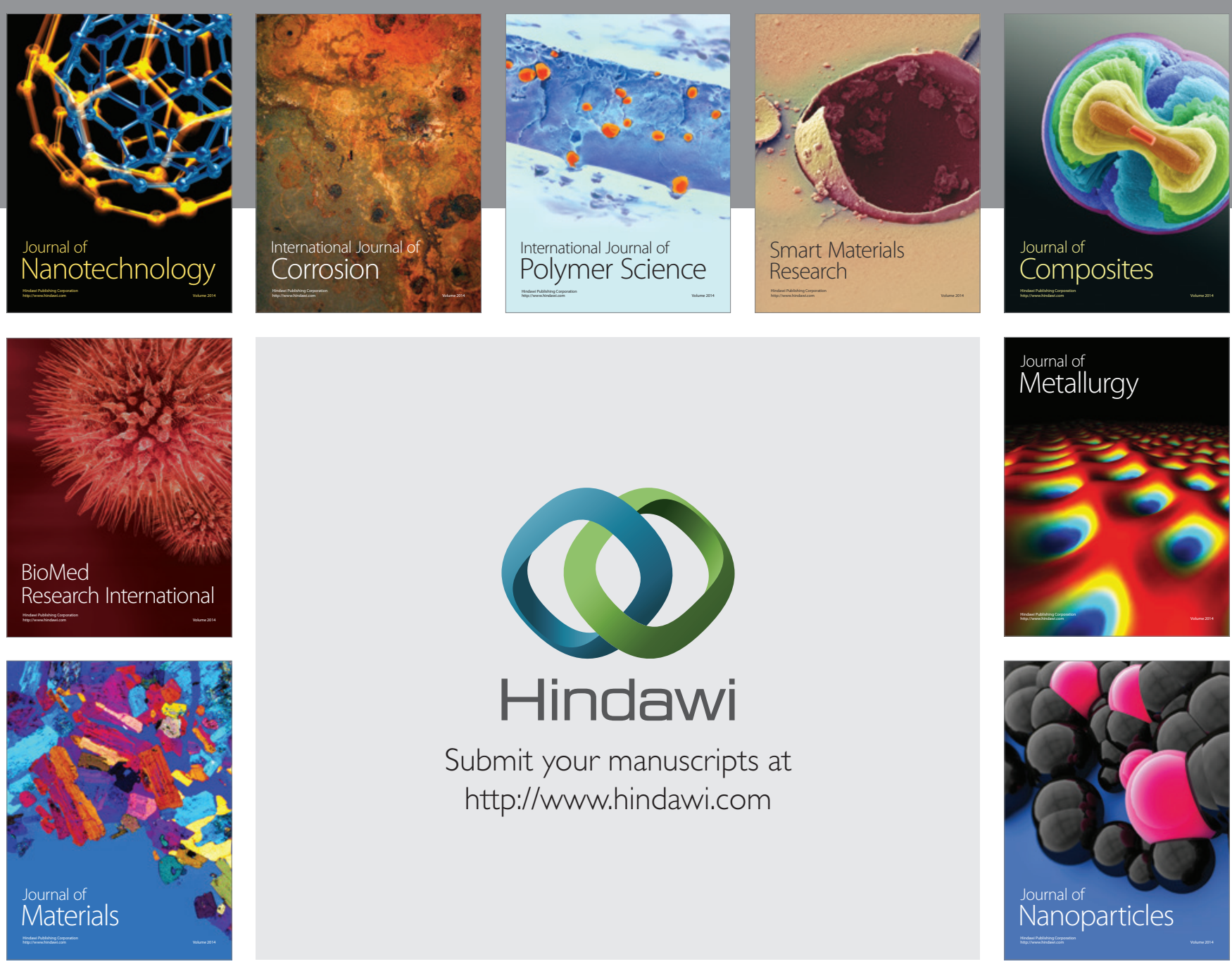

Submit your manuscripts at http://www.hindawi.com
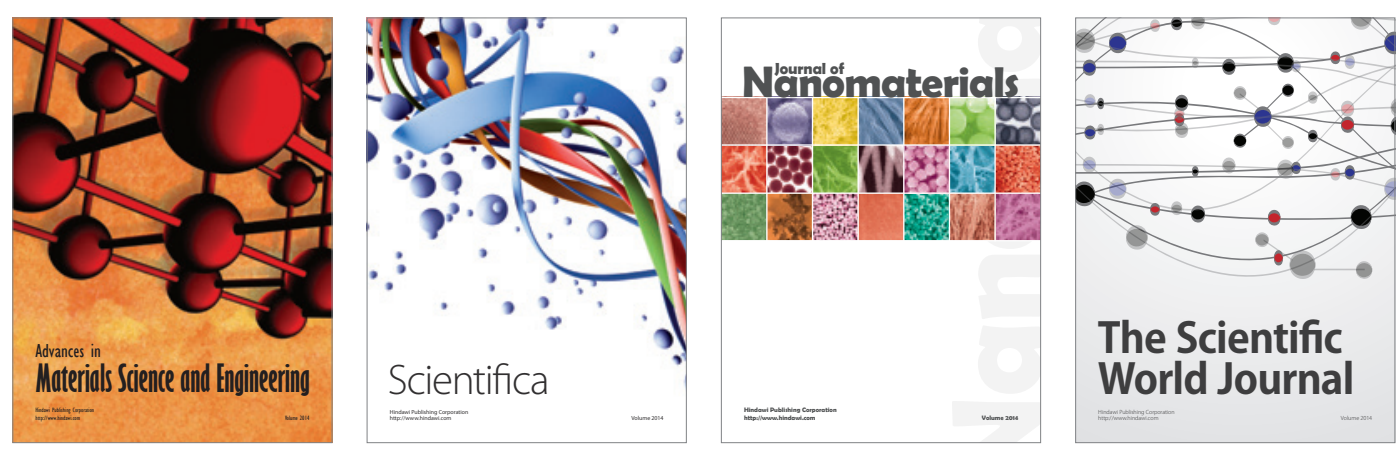

\section{The Scientific World Journal}
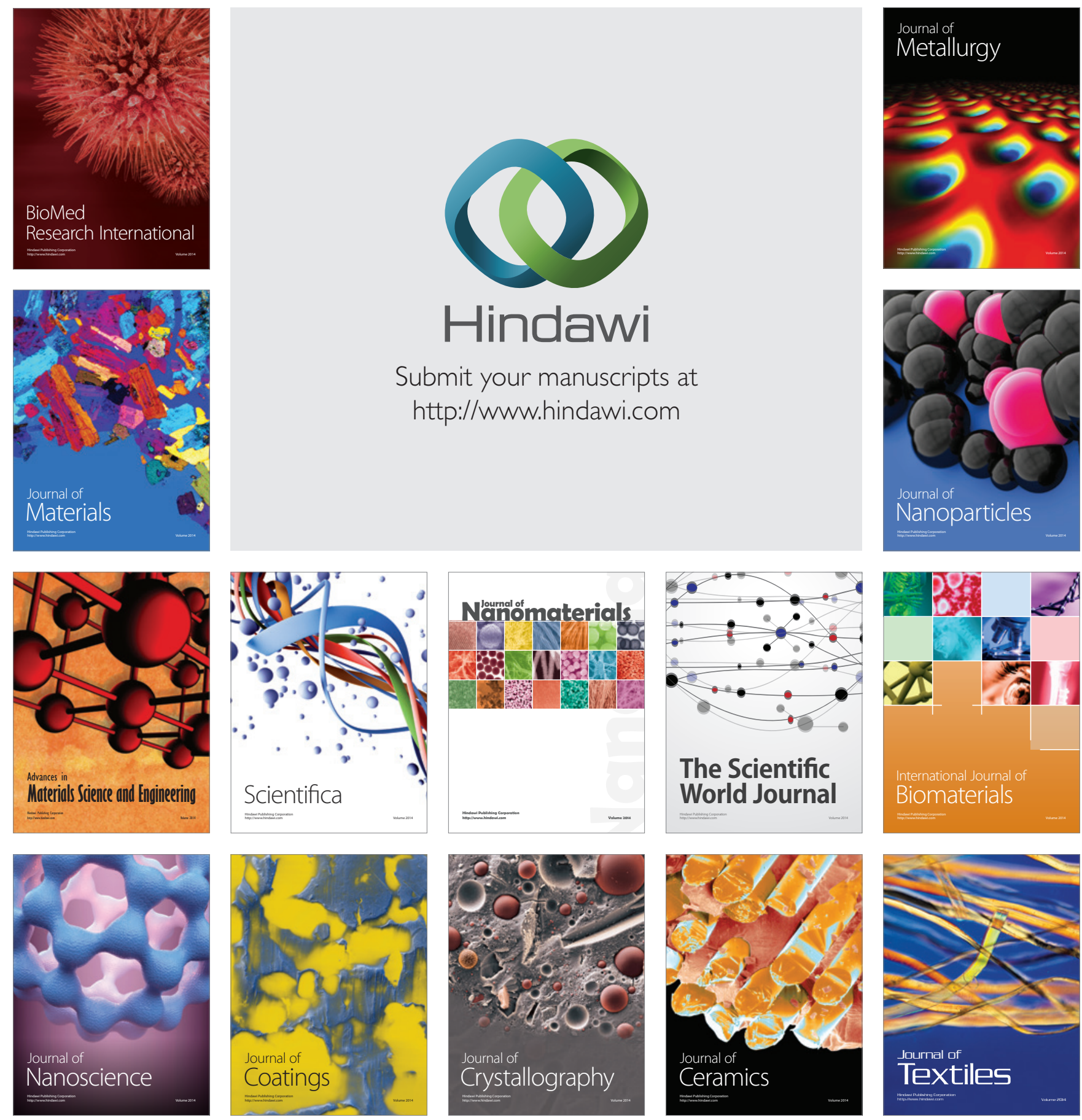